\title{
Parallel FM Signal Demodulation System based on Software Radio Platform
}

\author{
Yun Lin ${ }^{1 *}$, Jiaxing Wang ${ }^{1}$, Chao $\mathrm{Lv}^{1}$, Zhiqiang $\mathrm{Wu}^{2}$, Qiang $\mathrm{Li}^{2}$ and Zhiping \\ Zhang $^{2}$ \\ College of Information and Communication Engineering \\ Harbin Engineering University, Harbin, China \\ linyun@hrbeu.edu.cn \\ Department of Electrical Engineering \\ Wright State University, Dayton, Ohio, U.S. \\ zhiqiang.wu@wright.edu
}

\begin{abstract}
A parallel FM signal demodulation system is designed in this paper. All FM signals from $90 \mathrm{MHz}$ to $110 \mathrm{MHz}$ could be captured, separated and restored into original information. The method of digital channelized receiving is used in the system to divide the broadband signals into independent ones according to their center frequency. This design could be used in the field of electron reconnaissance and multichannel communication system. We realized the design on a software radio platform based on the structure of SCA (Software Communication Architecture), and the advantages of this ideal is discussed in the paper.
\end{abstract}

Keywords: software defined radio; digital channelized receiving; Software Communication Architecture; FPGA

\section{Introduction}

The frequency modulation (FM) is an important basic communication technology. It's used widely in the field of broadcast, amateur radio, space communication, etc. With the development of times, in some situation, the traditional single channel receiver couldn't satisfy the need of users, such as the whole frequency monitoring for policeman.

It is the primary idea of software defined radio to build an opening, standard, modularized general hardware platform[1]. More specific function will be realized by the software coding, making the communication system free from the limits of hardware structure. It's convenient and costs little to change or update the function of the system. The compatibility and connectivity between different systems will be also improved.

Software Communication Architecture (SCA) is a standard for software defined radio. It standardizes the software architecture, the hardware architecture, the security architecture, as well as the API interface specification for the software defined radio. It further enhances the portability, reusability, modularity and interoperability of system. Meanwhile, it illustrates the interaction between radio hardware and software and provides a software command set to control the components in system.

In this paper, a design is presented to receive all the broadcast FM signals, separate the signals into independent ones and recover the signals into original information. The method of digital channelized receiving and carrier frequency estimating is used in the design. The design is realized on a software radio platform based on the standard of SCA. Hardware resource is managed and called remotely by building a suitable SCA model, and the work of operation environment of SCA. 


\section{The Overview of System}

\subsection{Software Radio Platform based on SCA}

SCA (Software Communication Architecture) is formulated by CORBA software radio protocol and corresponding technical standards. It consists of Operating Environment and application. The Operating Environment includes Core Framework, CORBA middleware, embedded real-time operating system, network interface layer, and board support package[2]. The Application is a specific realization of software radio function, thus the design of specific software radio function could be separated from the physical layer.

As shown in Figure 1, it is our software radio platform. It consists of independent configuration center and equipment chassis. Configuration center is a computer with SCA Operating Environment. Equipment chassis is composed of controlling board, signal processing board, and radio frequency board. Controlling board initializes and updates the signal processing board and frequency board through the CPCI-bus, and connect with the configuration center through the network. Controlling board also equipped with an SCA Environment Operation, users can use the configuration center to load different applications into the Equipment chassis.

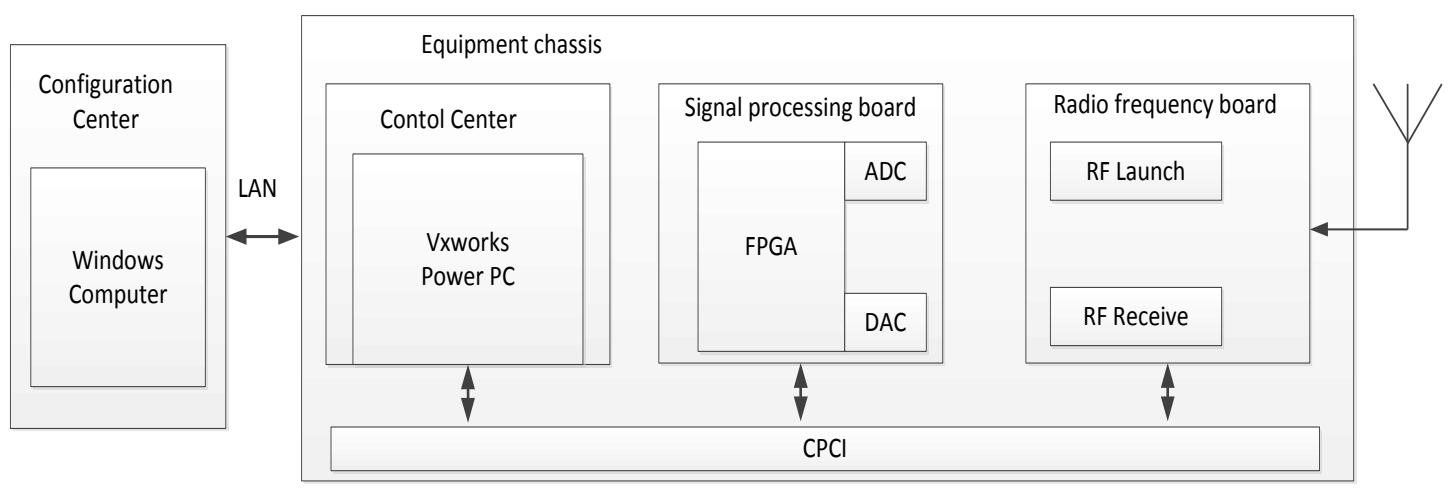

Figure 1. The Hardware Resource of Platform

\subsection{Application of Parallel FM Demodulation}

An application of parallel FM signal demodulation is designed in this paper. The application aims to capture, separate and recover all the FM signals with the frequency from $90 \mathrm{MHz}$ to $110 \mathrm{MHz}$.

As shown in Figure 2 is the overview of the application. All the signals from $90 \mathrm{MHz}$ to $110 \mathrm{MHz}$ are received by the antenna. Then they are amplified and filtered by the radio frequency board. The suitable signals will be sampled by the ADC with the speed $240 \mathrm{MHz}$, then digital down converter algorithm program in FPGA changes the signal into zero intermediate frequency signals, the form of the signal is converted into complex signal from real signal. For the effective bandwidth is only $20 \mathrm{MHz}$, the data could be extracted by 12 times to reduce the redundant. To demodulate the signals, effective separation is necessary. The algorithm of digital channelized is used to divide the signals into independent sub-channels, according to their center frequency. In our design we divide them into 64 sub-channels. There exists no signal in some channels. Channel decision method based on the energy is also needed to choose valid sub-channels. Carrier frequency is estimated before demodulation. Finally, the information of signals would be sent to the users through the network. And the voice and waveform is played in the configuration computer. 


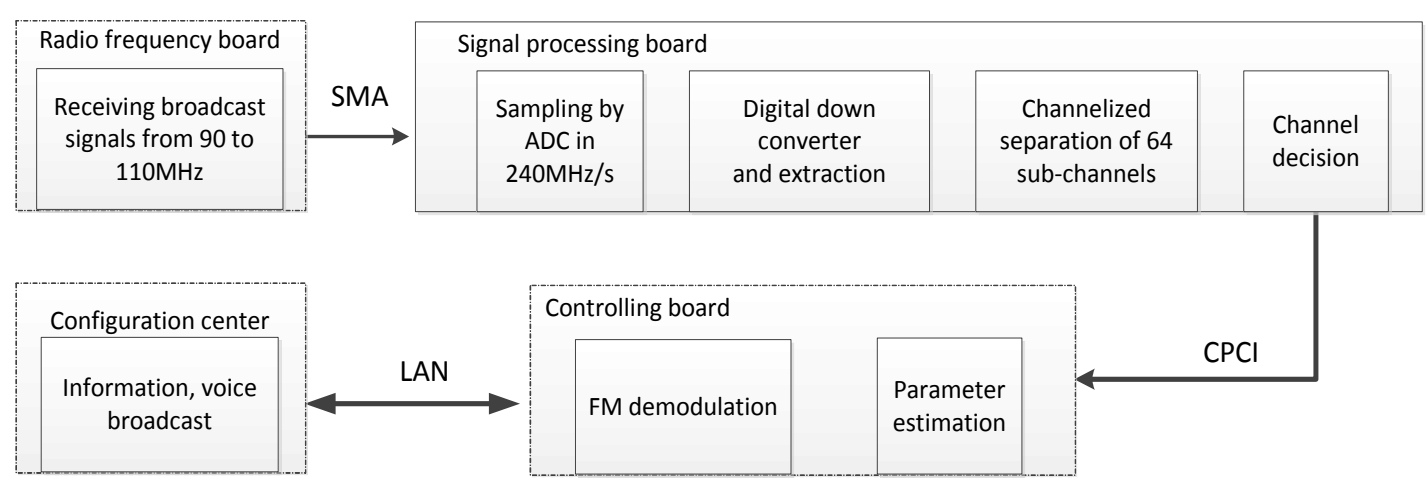

Figure 2. The Overview of Application of Parallel FM Demodulation

\section{Separation of Signals}

To demodulate all signals of $20 \mathrm{MHz}$, the separation of signals is the foundation. Therefore, algorithm of digital channelized receiving is used in the application.

\subsection{Abstract of Digital Channelized Receiving}

As one of the key technologies of the broadband digital receiver and software radio system, the algorithm of digital channelized receiving is used to extract single or multiple independent sub-band signals from wideband mixed signals [3]. The implementation of specific functions include: digital down frequency conversion, filtering, sampling rate conversion, etc.

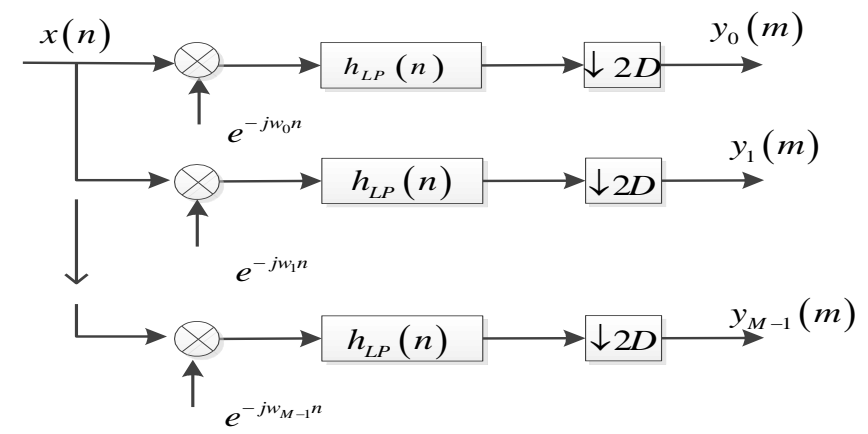

Figure 3. The Basic Model of the Digital Channelized Receiver

Shown as the Figure 3, it is a basic model of digital channelized receiving. $x(n)$ is the broadband signal that containing many signals. It enters into different channels where different local digital oscillators. The amount of channels is M. The signal $x(n)$ mixes with these different local digital oscillators. Different frequency components in $x(n)$ would be mixed into low-pass signals. Then an original low-pass filter removed the high frequency part in each channel. The bandwidth of the original low-pass filter is one of $\mathrm{M}$ points to the whole sampling rate. Finally, different frequency components are gotten in different channels. In each of the channels, the valid frequency parts are narrow to the whole frequency band. According to the decimation theory of digital signal processing, M times extraction could be made to reduce the data amount. The outputs of each channel are the different frequency components in the broad- band signal $x(n)$. When the amount of channels is many enough, every independent sub-signal could be separated thoroughly.

\subsection{Efficient Structure of the Digital Channelized Receiving}

In 1987, Vaidyanathan put forward the theory of $M$ channels filter bank[4]. In fact, the algorithm of digital channelized receiving is one of key applications of the theory. Today, 
it plays an important role in the field of software radio. Corresponding studies are also heated. From the Figure 3, we find that it costs much hardware resource such as the multi-pier, storage unit and so on to mix and filter the signal. Therefore, how to reduce the calculation amount becomes an important and difficult issue in the field of communication. Here, an efficient structure is introduced in the paper.

Shown as Figure 3, the frequency band is $1 / M$ of the whole bandwidth. When $x(n)$ is a complex signal, the division of frequency bands of channels could be shown as the Figure 4.

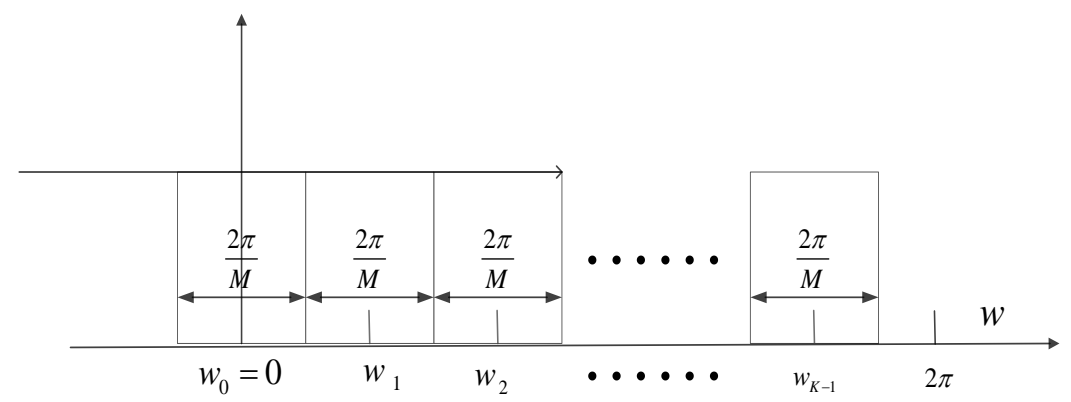

\section{Figure 4. The Division of Channel of the Complex Signal}

Center frequency of each channel is as in (1)

$w_{k}=2 \pi k / M \quad k=0,1, M-1$

Here we use the method of ploy-phase decomposition. The output of number k subchannel is given as:

$$
\begin{aligned}
y_{k}(m) & =\left.\left[x(n) e^{j w_{k} n}\right] * h_{L P}(n)\right|_{n=m D} \\
& =\sum_{i=0}^{N-1} x(m D-i) e^{j w_{k}(m D-i)} h_{L P}(i) \\
& =\sum_{p=0}^{M-1} \sum_{i=0}^{\frac{N}{M}-1} x(m D-i M-p) e^{j w_{k}(m D-i K-p)} h_{L P}(i M+p)
\end{aligned}
$$

The $y_{k}(m)$ is the output of number $\mathrm{k}$ sub-channel, $k=0,1, \cdots M-1, x(n)$ is the input of the system, $w_{k}$ is the centre frequency of the number k sub-channel, $h_{L P}(n)$ is a low-pass filter with a cut-off frequency of $\pi / M, M$ is total number of the sub-channel, $N$ is the filter order of $h_{L P}(n)$.The $D$ is the decimation times of the signal, its value is equal to $K / F, F$ is a positive integer[5]. Then we decompose the signal and filter into the poly-phase [6] form as following.

$$
\begin{gathered}
x_{p}(m)=x(m D-p) \\
g_{p}(m)=h_{L P}(m K+p), p=0,1 \cdots M-1
\end{gathered}
$$

We can get

$$
\begin{aligned}
y_{k}(m) & =\sum_{p=0}^{M-1}\left[\sum_{i=0}^{L-1} x_{p}(m-i F) e^{j w_{k}(m-i F) D} g_{p}(i)\right] e^{-j w_{k} p} \\
& =\sum_{p=0}^{M-1}\left[\sum_{i=0}^{(L-1) F} x_{p}(m-l) e^{j w_{k}(m-l) D} g_{p}\left(\frac{l}{F}\right)\right] e^{-j w_{k} p}
\end{aligned}
$$


In the above formula, the $M=D F, L=\frac{N}{M}$, then we define $g^{\prime}{ }_{p}(l)=g_{p}\left(\frac{l}{F}\right)$, the $g^{\prime}{ }_{p}(l)$ can be got by interpolating the $g_{p}(l) \mathrm{F}$ times. We could further get

$y_{k}(m)=\sum_{p=0}^{M-1}\left\{\left[x_{p}(m) e^{j w_{k} m D}\right] * g_{p}^{\prime}(m)\right\} \cdot e^{-j w_{k} p}, \quad k=0,1, \cdots, M-1$

Then we define $x_{p}^{\prime}(m)=\left[x_{p}(m) e^{j w_{k} m D}\right] * g^{\prime}{ }_{p}(m)$, we can finally get

$y_{k}(m)=\sum_{p=0}^{K-1} x_{p}^{\prime}(m) e^{-j w_{k} p}=\operatorname{DFT}\left(x_{p}^{\prime}(m)\right)$

If $F=2$, we could design a digital channelized structure as the Figure 5 .

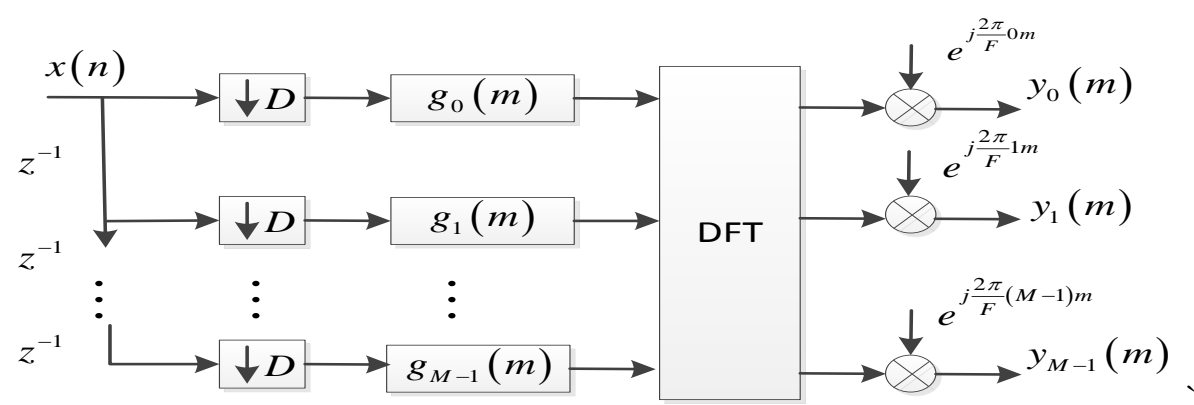

Figure 5. The Efficient Structure of the Channelize Receiver

In the structure as Figure 5, the storage units that deposits the filter coefficients are reduced $\mathrm{D}$ times. The computation of filtering signal is reduced $D^{2}$ times.

In order to separate the independent signal, the amount of channels needs to be determined. In our design, the minimum frequency interval $G_{\min }$ and the whole frequency band $B_{w}$ is concerned. In Harbin (a city in Heilongjiang Province in China) region, the frequency of FM broadcast is shown in the Table 1.

\section{Table 1. The Center Frequency of FM Broadcast In Harbin}

\begin{tabular}{|c|c|}
\hline Center frequency & Name of the Broadcast Radio \\
\hline $94.6 \mathrm{MHz}$ & The News of Hei Longjiang \\
\hline $95.8 \mathrm{MHz}$ & The Music of Hei Longjiang \\
\hline $97.6 \mathrm{MHz}$ & FM 97 of Hei Longjiang \\
\hline $98.4 \mathrm{MHz}$ & Literature of Harbin \\
\hline $99.3 \mathrm{MHz}$ & $\begin{array}{c}\text { Colleges and Universities of Hei } \\
\text { Longjiang }\end{array}$ \\
\hline $99.8 \mathrm{MHz}$ & Traffic of Hei Longjiang \\
\hline $100.9 \mathrm{MHz}$ & The Sound of Music \\
\hline $103.5 \mathrm{MHz}$ & Women of Hei Longjiang \\
\hline $104.5 \mathrm{MHz}$ & The City life of Hei Longjiang \\
\hline $105.6 \mathrm{MHz}$ & Peopele's broadcasting of Harbin \\
\hline
\end{tabular}

From the Table 1, we known the minimum frequency interval $G_{\min }=99.8 \mathrm{MHz}-99.3$ $=0.5 \mathrm{MH}_{3}$. The whole frequency band $B_{w}=20 \mathrm{MHz}$. The number should be $N u m \geq \frac{D_{w}}{{ }^{2}}=40$, when the Num is equal to $2^{n}$, the structure is easy to realize, so we designed $G_{n} \emptyset_{4}$ sub-channels digital channelized receiving structure.

\subsection{The Way to Get the Complex Signal $x(n)$}

From the content above, we known the efficient structure of digital channelized receiving as Figure 5 could deal with the complex signal only. However, the signal we 
got from $\mathrm{ADC}$ is the real signal from $90 \mathrm{MHz}$ to $110 \mathrm{MHz}$. A step of digital down conversion as following is need[7]:

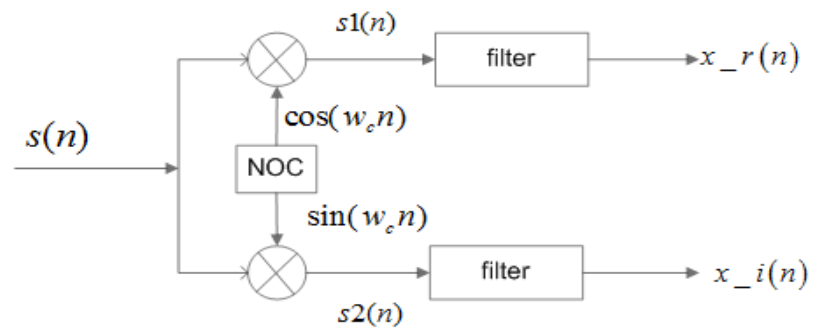

Figure 6. The Model of Digital Down Conversion

At time $\mathrm{t}$, the input signal that with the frequency from $90 \mathrm{MHz}$ to $110 \mathrm{MHz}$ is as formula

$$
s(n)=x(n) \cos \left(w_{c} n+\varphi\right)
$$

The $x(n)$ is the amplitude information of $s(n)$, the $\varphi$ is its phase information, $w_{c}$ is its carrier frequency, here it's equal to $100 \mathrm{MHz}$. The input $s(n)$ will be mixed by a couple of orthogonal local carrier frequency $\cos \left(w_{c} n\right)$ and $\sin \left(w_{c} n\right)$. We can get

$$
\begin{aligned}
& s 1(n)=x(n)\left(\cos \left(2 w_{c} n+\varphi\right)+\cos (\varphi)\right) \\
& s 2(n)=x(n)\left(\sin \left(2 w_{c} n+\varphi\right)-\sin (\varphi)\right)
\end{aligned}
$$

Then above two signals will pass a filter to remove the high-frequency component. This filter is combination of CIC filter and fir filter. Finally we got a couple of orthogonal component of the $s(n)$. They are as following:

$$
\begin{aligned}
& x_{-} r(n)=x(n) \cos (\varphi) \\
& x_{-} i(n)=-x(n) \sin (\varphi)
\end{aligned}
$$

Use this couple of orthogonal component we could change the real signal $s(n)$ into a complex signal $x(n)$.

$$
x(n)=x_{-} r(n)-x_{-} i(n) \times i
$$

\subsection{Verification of Signal Separation}

To verify the effect of separation of FM signal, we took a test according to the description of above. And we got the result of outputs of 64 sub-channels shown as the Figure 7. There exists only one FM signal in each channel.

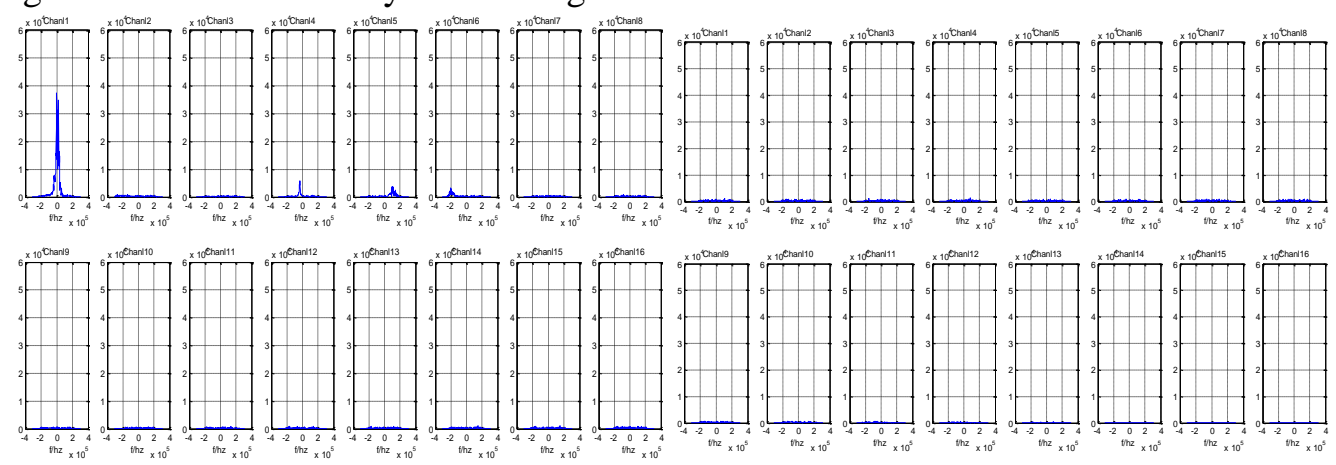



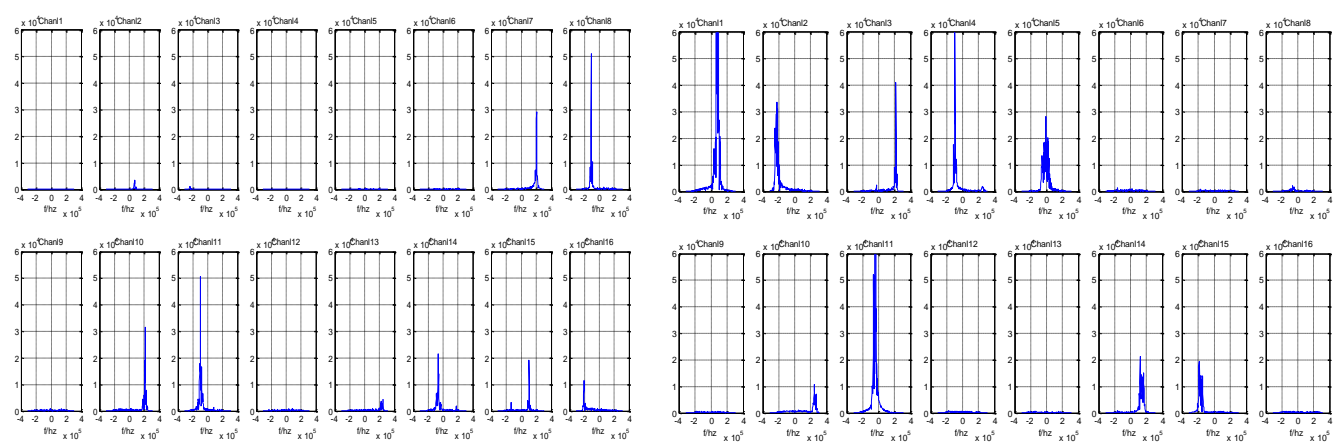

Figure 7. Separation of Signals

\section{Information Recover}

\subsection{Decision of Channels with Valid Signal}

From the Figure 7, we know that there exists no signal in many sub-channels. Meanwhile, the original filter in the digital channelized receiving will bring spectrum aliasing. Efficient method is necessary to choose the channels contain valid signals [8]. Here the method of energy decision in the frequency domain is used.

Primarily, we calculate the discrete fourier transform of each output of the subchannel.

$y_{n}(k)=F F T\left(y_{n}(m)\right) \quad n=0,1,2 \cdots M-1$

In our design, we did 1024 points FFT. From 257 to 768 points are valid frequency points, according to the $50 \%$ aliasing original filter. According to the Parseval's theorem, the energy of sub-channel could be obtained by integral the valid spectrum component.

$E_{y_{n}(k)}=\sum_{257}^{768} y_{n}(k) \quad n=0,1,2 \cdots M-1$

The energy of sub-channel with signal is equal to energy of signal added the energy of noise. It's larger than the energy of sub-channel only containing the noise. The average energy of the whole frequency band is calculated as the decision threshold $E_{\text {avg }}$. We compared all the energy of each channel with the $E_{\text {avg }}$, and find the sub-channels which contain valid signal. The result is shown as following.

Sub-channels contain valid FM signal are 1,39,40,43,49,50,51,52,53,59

\subsection{Estimation of Carrier and Demodulation of Signal}

To demodulate the signals, it's necessary to remove high frequency carrier[9]. However, the frequency of FM (frequency modulation) is changeable, according to the modulated content. Here, a method to calculate the geometry center of gravity[10] is used to get their center carrier.

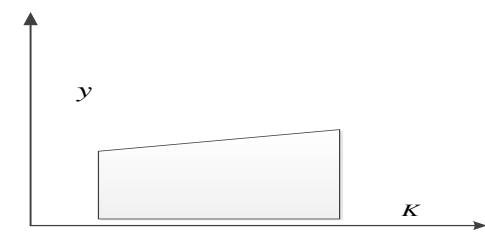

Figure 8. Geometric Shape in Coordinate 
Like the Geometric shape in coordinate, the abscissa of center $I=\frac{\sum k y^{2}}{\sum y^{2}}$.

The frequency domain data $y_{n}(k)$ of sub-channel could be regard as a Geometric shape in coordinate, the frequency is abscissa, the strength is ordinate. And the center frequency

$$
F_{c}=\frac{\sum k y_{n}^{2}(k)}{\sum y_{n}{ }^{2}(k)}
$$

Using this method, we estimated the carrier frequency of these signals, the comparison is shown as Tab.2.

Table 2. The Estimation Result of Carrier Frequency

\begin{tabular}{ccc}
\hline $\begin{array}{c}\text { Original carrier } \\
\text { frequency }\end{array}$ & The estimation result & Error \\
\hline $94.6 \mathrm{MHz}$ & 94.57 & $0.03 \%$ \\
$95.8 \mathrm{MHz}$ & 95.75 & $0.05 \%$ \\
$97.6 \mathrm{MHz}$ & 97.54 & $0.06 \%$ \\
$98.4 \mathrm{MHz}$ & 98.40 & $0.00 \%$ \\
$99.3 \mathrm{MHz}$ & 99.27 & $0.03 \%$ \\
$99.8 \mathrm{MHz}$ & 99.81 & $0.01 \%$ \\
$100.9 \mathrm{MHz}$ & 100.87 & $0.03 \%$ \\
$103.5 \mathrm{MHz}$ & 103.52 & $0.02 \%$ \\
$104.5 \mathrm{MHz}$ & 104.51 & $0.01 \%$ \\
$105.6 \mathrm{MHz}$ & 105.62 & $0.02 \%$ \\
\hline
\end{tabular}

The carrier frequency would be removed based on the value estimated. The envelope of signal would be demodulated by discriminating the frequency.
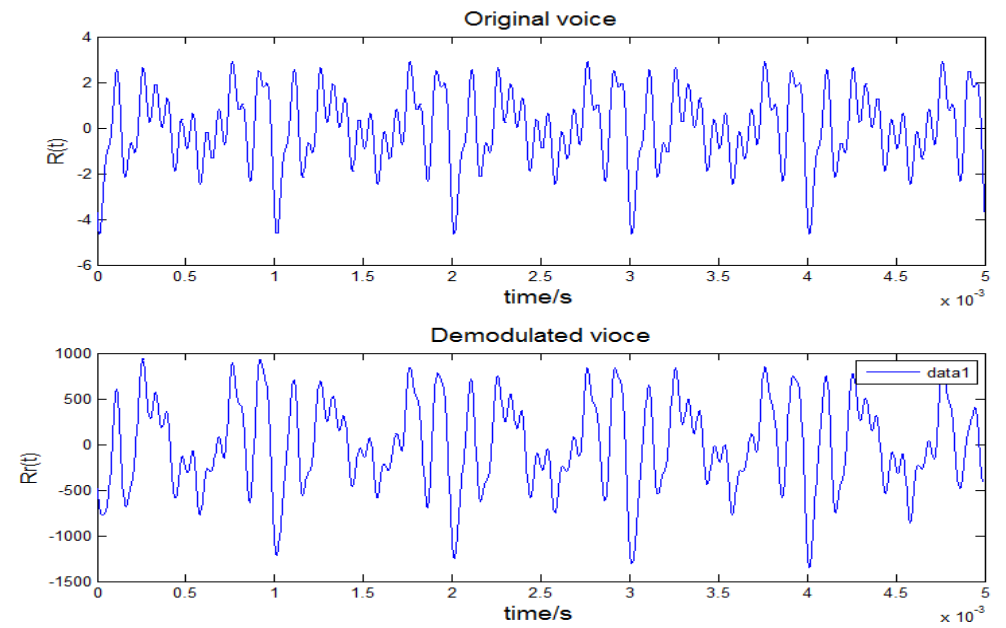

Figure 9. Comparison between Testing Voice and Demodulated Voice

We generated the FM signal with kwon waveform and sent it into the system and extract the testing data, it's shown as 


\subsection{Modeling of SCA}

From the Figure 1, we known the platform we used is based on the standard of SCA (Software Communication Architecture). In this platform the way of loading executive file(.out/bit) into hardware to change the function of platform is replaced by using the middle component of SCA to call the hardware resource.

In our platform, the operation system of vxworks5.5.1 and the SCA operation environment is installed on the chip PowerPC in controlling board. The application model of parallel FM demodulation is compiled into XML file and .out file by the tool software Spectra CX. Part of the model is shown as the Figure 10.

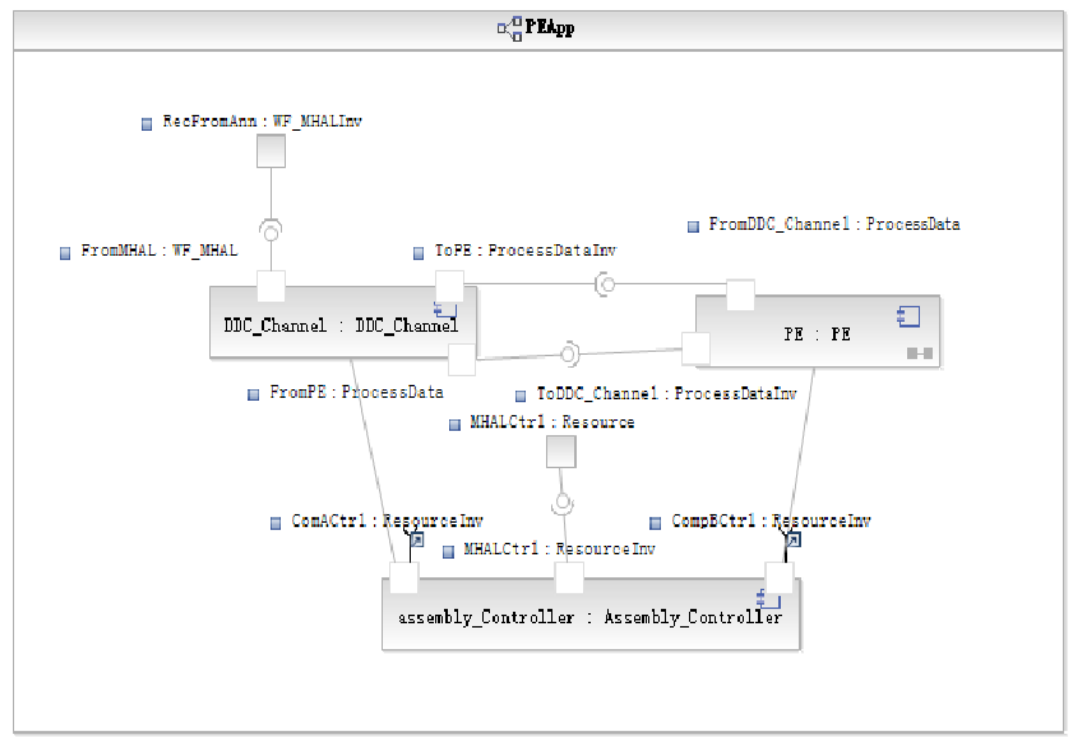

Figure 10. SCA Application Model of Parallel FM

In the model the DDC_Channel is the component of digital down convert and digital channelized receiving; the PE is the component of frequency parameter estimation; the assembly_Controller is the component of assembly and management of hardware resource. By using the model, a application program library is generated. In the program library, we added the distribution of hardware resource for the function component, like is FPGA is used to achieve the DDC_Channel, the PowerPC is used to achieve PE .Then the model is compiled into XML files and out files. These files will be saved in the computer for user.

When we need load the application of parallel FM demodulation, we connect the controlling board using the network at first. Then we load the XML and out and bit files of the model into the chip PowerPC in controlling board. The model will be launched in the SCA operated environment. All the hardware resources would be initialized and controlled by the model. The frequency board would receive the signals from $90 \mathrm{MHz}$ to $110 \mathrm{MHz}$, after sampling the signals with ADC the FPGA would remove center frequency of signals and separate the signals into independent ones. The data in the sub-channels that contained valid signal would be sent into the controlling board. Carrier frequency of every signal is estimated in the PowerPC. Finally the data would be sent into the configure computer and be demodulated in the program in computer.

\subsection{Performance of System}

We made a measurement for our application to testing its performance. The instrument we used is our equipment, a single frequency modulation receiver Tecsum-A3, the place we made the test is Harbin in China. We started our 
equipment, loaded the application into the software platform and received all the ten waveforms. The waveforms of voices are shown in the Figure 11. And we listened the sounds played by our equipment and compared them with the voices played by Tecsum-A3, the contents are accordance.

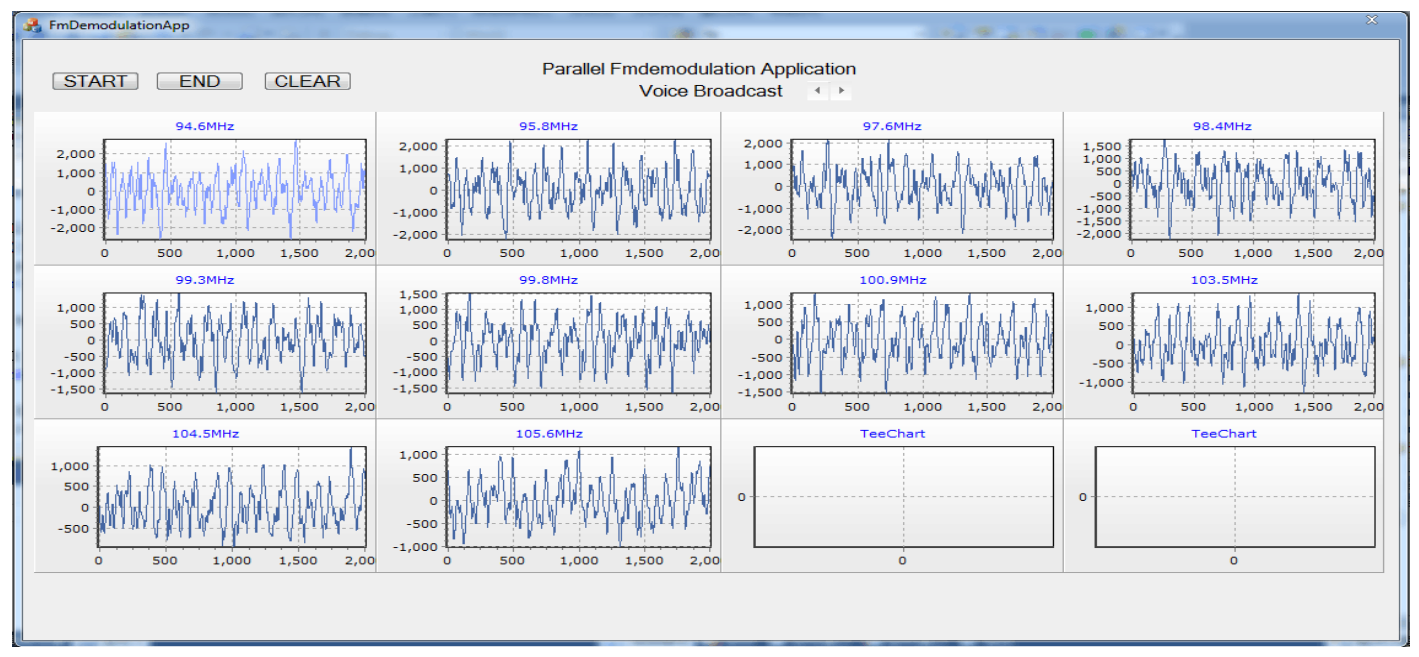

Figure 11. Waveforms of FM broadcast signals

\section{Conclusion}

In this paper, we introduced the software platform based on the standard of SCA, and the work of SCA. An application of parallel FM demodulation is presented. All the FM signals from $90 \mathrm{MHz}$ to $110 \mathrm{MHz}$ could be received, separated, and demodulated into original voice information. Corresponding algorithms and distribution of hardware resource in the application is analyzed. Finally, a test is made to test the performance of system. All ten broadcast signals are recovered into the waveforms and voice. Comparison with one kind of traditional FM receiver Tecsum-A3 is made. The contents are same, but the quality of voice is a little worse than the Tecsum-A3. Parallel demodulation is our advantage, but more step bring more noise, the elimination of noise in the system could be our research question in the future.

\section{Acknowledgment}

This work was supported by the Key Development Program of Basic Research of China(JCKY2013604B001), the Nation Nature Science Foundation of China (No.61301095 and No.61201237), Nature Science Foundation of Heilongjiang Province of China (No. QC2012C069 and F201408) and the Fundamental Research Funds for the Central Universities (No. HEUCF1508).

Meantime, all the authors declare that there is no conflict of interests regarding the publication of this article.

\section{References}

[1] J. Mitola and G. Q. Maguire, "J. Cognitive radio: making software radios more Personal", IEEE Personal Communication, vol. 3, no. 15, (1999).

[2] S. Jia, Y. Hada, G. Ye, K. Takase, "Remote Control for Robotic Systems Using CORBA as Communication Architecture", Remote Control for Robotic Systems Using CORBA as Communication Architecture, vol. 1, no. 2, (2002).

[3] Y. Tian-feng, Q. Zhong-wei and S. Pei-lan, "J. Integrated Channelization Receiver System of SDR", Communications Technology, vol. 4, no. 42, (2009).

[4] P. P. Vaidyanathan, "J. Theory and design of $\mathrm{M}$ channel maximally decimated quadrature mirror filters with arbitrary M", having the perfect-reconstruction property. IEEE Trans. ASSP, vol. 4, no. 35, (1987). 
[5] K. Tanja and N. J. Fliege, "J. Modified DFT filter banks and perfect reconstruction", IEEE Transactions on circuits and system, vol. 6 , no. 46, (1999).

[6] X. Zhou, "Digital signal processing for coherent multi-level modulation formats", Chinese Optics Letters, vol. 2, no. 9, (2010).

[7] J. Fudge, L. Mand Sehreiner C., "An approach to efficient wideband digital down conversion", In: Proc. Of ICSPAI, Toronto, (1998), pp. 713-717.

[8] S. Dan, G. Lin-dong and Q. Dan, "A Novel Presence Detection Algorithm Based on the Energy Detection for Burst Signals[J]", Single Processing, vol. 24, no. 4, (2008).

[9] P. O'shea, "J. A fast algorithm for estimating the parameters of a quadratic FM Signal", IEEE Transaction on Signal Processing, vol. 5, no. 52, (2004).

[10] Z. Zhao, M. Pan and Y. Chen, "J. Instantaneous frequency estimate for non-stationary signal", Intelligent Control and Automation, Wcica, Fifth World Congress on, vol. 1, no. 4, (2004). 
International Journal of Signal Processing, Image Processing and Pattern Recognition Vol.9, No.3 (2016) 